\title{
Probing Defects and Impurity-induced Electronic Structure Changes in Single and Double-layer Hexagonal Boron Nitride Sheets with STEM-EELS
}

\author{
Q.M. Ramasse ${ }^{1}$, N. Alem ${ }^{2}$, O.V. Yazyev ${ }^{3}$, A. Zettl ${ }^{2}$, C.T. Pan ${ }^{4}$, R.R. Nair ${ }^{4}$, R. Jalil ${ }^{4}$, R. Zan ${ }^{4,5}$, U. \\ Bangert $^{5}$, C.R. Seabourne ${ }^{6}$, A.J. Scott ${ }^{6}$ and K.S. Novoselov ${ }^{5}$
}

${ }^{1}$ SuperSTEM Laboratory, STFC Daresbury, Keckwick Lane, Daresbury WA4 4AD, U.K.
${ }^{2}$ Department of Physics, University of California - Berkeley, Berkeley CA94720
${ }^{3}$ Institute of Theoretical Physics, EPFL, CH-1015 Lausanne, Switzerland
${ }^{4}$ School of Materials, The University of Manchester, Manchester M13 9PL, U.K.
${ }^{5}$ School of Physics and Astronomy, The University of Manchester, Manchester M13 9PL, U.K.
${ }^{6}$ Institute for Materials Research, SPEME, University of Leeds, Leeds LS2 9JT, U.K.

While graphene is rapidly moving from the laboratory to the factory floor, a sustained research effort has been applied to studying how it interfaces with the other components of emerging graphenebased electronic devices [1]. As a result, other two-dimensional materials with structures analogous to graphene but with complementary properties, have attracted renewed attention [2]. Single-layer hexagonal boron nitride (h-BN) is one such material whose wide band gap of $\sim 5.9 \mathrm{eV}$ arguably makes it the thinnest possible insulator [3]. Impurities, defects or structural tailoring have however the potential to dramatically modify the electronic structure and therefore the properties of these twodimensional crystal membranes. The recent advances in atom-by-atom chemical analysis using annular dark field scanning transmission electron microscopy [4] provide the perfect tool to image directly defects or edge structures in h-BN sheets, or to identify unambiguously impurities within the lattice. We combine here Z-contrast STEM imaging at $60 \mathrm{kV}$ acceleration voltage on a Nion UltraSTEM100 with electron energy loss spectroscopy (EELS) in both low- and core-loss regimes to probe defect-induced changes in the electronic structure of single and double layer h-BN.

As is the case for graphene, the plasmon structure of single and bi-layer h-BN is quite unique: fig. 1a. While these valence EELS results generally agree with ab initio DFT simulations carried out with WIEN2k, the detailed shape of the calculated $\pi$-plasmon for pure h-BN shows slight discrepancies with experimental spectra. In particular, a shoulder systematically present at $\sim 7.5 \mathrm{eV}$ in the experiments is not reproduced by simulations. Careful image analysis reveals the integration within the h$\mathrm{BN}$ lattice of about $4 \%$ of $\mathrm{O}$ and $\mathrm{C}$ atoms, as single $\mathrm{O}$ atoms or $\mathrm{C}-\mathrm{C} / \mathrm{C}-\mathrm{O}$ short chains (rather than clusters): fig. $1 \mathrm{~b}$. The incorporation in the corresponding amounts of these impurities into large supercells for further WIEN2K simulations leads to a much improved agreement with the experiments (fig. 1c) and confirms the large influence of $\mathrm{O}$ impurities on the electronic structure of h-BN [5].

Similarly, lattice relaxations occurring at the edges of a bi-layer h-BN sheet lead to noticeable electronic structure changes. Rather than staying flat, the edges fold and bond together: fig. 2a-b. This reconstruction is obvious through the strain-induced contrast in ADF images, also observed in simulated images of the relaxed structure calculated by DFT. Core-loss B $K$ EEL spectra acquired at specific atomic positions along and away from the folded edge show distinct EELS fine features, notably a change of the $\pi^{*}$ to $\sigma^{*}$ ratio, in extremely good agreement with ab initio simulations: fig. $2 \mathrm{c}$. Understanding such atomic scale chemical and physical effects in $2 \mathrm{D}$ crystal membranes will no doubt prove essential for nano-engineering attractive properties for these novel materials. 
References

[1] R. Zan et al., Nanoletters 11, 1087 (2011).

[2] K. Novoselov et al., Proc. Natl. Acad. Sci. USA 102, 10451 (2005).

[3] K. Watanabe et al., Nature Materials 3, 404 (2004).

[4] O.L. Krivanek et al., Nature 464, 572 (2010).

[5] C.T Pan et al., Phys. Rev. B 85, 045440 (2012).

[6] This work was supported in part by the EPSRC, US DOE under contract DEAC02-05CH11231 and the Center of Integrated Nanomechanical Systems with grant number EEC-0425914.

(a)

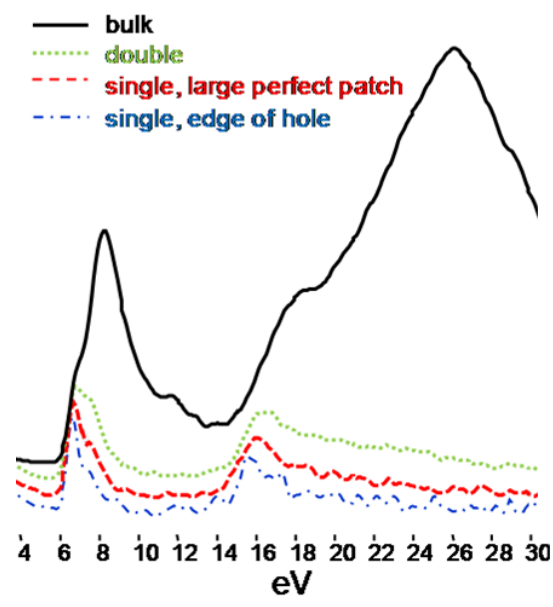

(b)

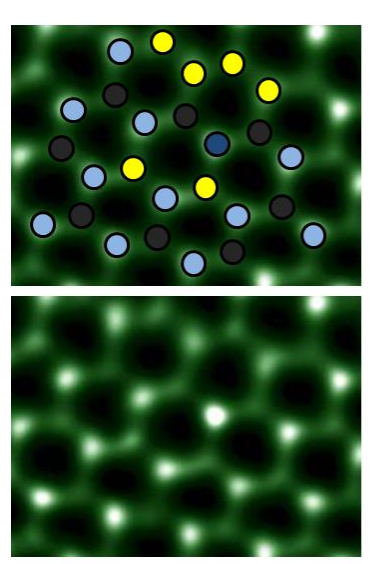

(c)

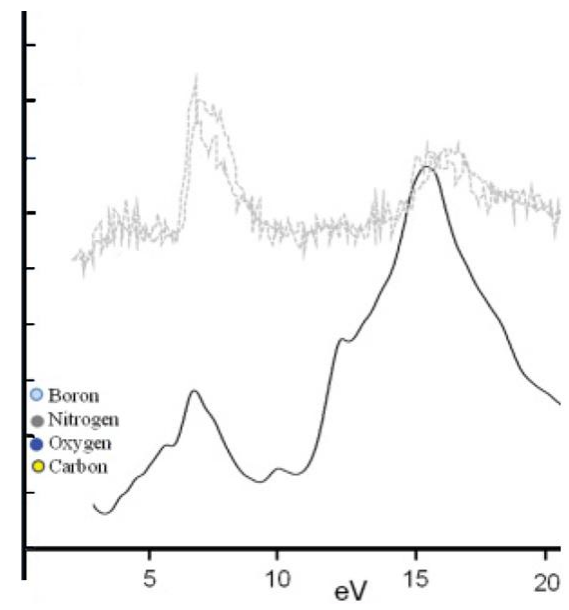

FIG. 1. (a) Experimental spectra from single layer (both from a large layer and in aloof mode at the edge of a hole), double layer and bulk (10+ layers) h-BN. A shoulder at $7.5 \mathrm{eV}$ is clearly visible on the $\pi$ plasmon (arrow) but is not reproduced in simulations of pure h-BN. (b) Histogram intensity analysis [4] of HAADF images reveals the presence of $\sim 4 \%$ of $\mathrm{C}$ and $\mathrm{O}$ impurities. (c) WIEN2k calculation of an EEL spectrum (solid black line) from a structure similar to that of (b), showing that the $7.5 \mathrm{eV}$ shoulder from experimental spectra (grey line above) is well reproduced. [5]

(a)

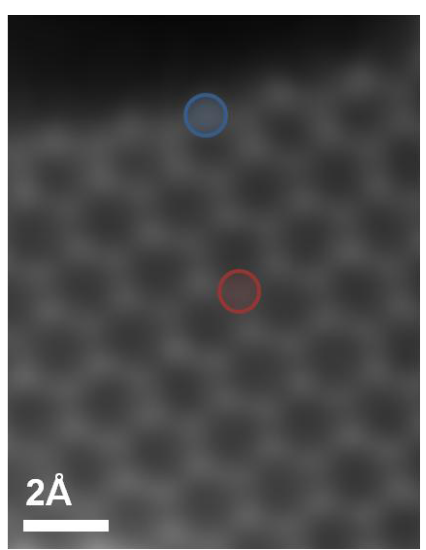

(b)

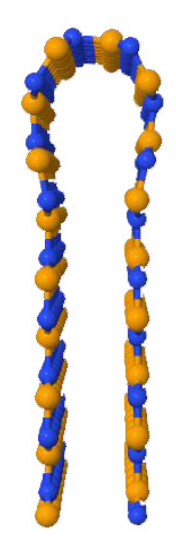

(c)

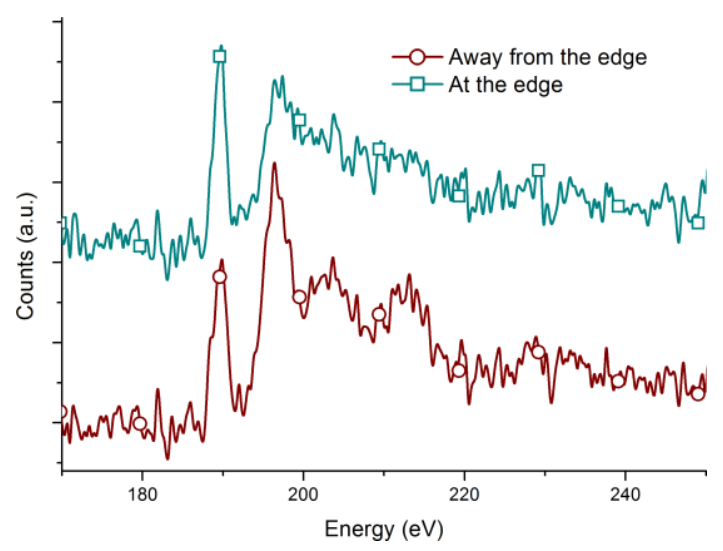

FIG. 2. (a) Low-pass filtered ADF image of the folded edge of a bi-layer h-BN sheet (the number of layers was identified through VEELS and by contrast analysis). The lattice relaxation at the edge results in strain-induced contrast. (b) Edge-on model of the folded sheet: the relaxed atomic positions were calculated by DFT. (c) B $K$ EELS spectra acquired at the positions marked in (a). Clear $\pi^{*}$ to $\sigma^{*}$ ratio differences can be observed, in agreement with ab initio WIEN2k calculations (not shown). 